\title{
CT attenuation correction for thallium SPECT MPI and other benefits of multimodality imaging
}

\author{
Randall C. Thompson, MD, FASNC ${ }^{\mathrm{a}}$ \\ a Saint Luke's Mid America Heart Institute and The University of Missouri-Kansas City, Kansas \\ City, MO
}

Received Mar 5, 2018; accepted Mar 5, 2018

doi: $10.1007 / \mathrm{s} 12350-018-1255-2$

\section{See related article, pp. pp. 1584-1595}

Far from being a static field, nuclear cardiology has enjoyed one advance after another during the last three decades. ECG gating for measurement of left ventricular ejection fraction and assessment left ventricular wall motion, attenuation correction, quantitation of perfusion defects, improvement in instrumentation with better collimators and detectors, advanced post processing including iterative reconstruction, and quantitation of myocardial blood flow have bestowed on this valuable medical tool ever greater levels of accuracy and applicability.

In this issue of the journal, Huang et al., have contributed to this trend by showing the value of CT attenuation for thallium SPECT MPI imaging. ${ }^{1}$ Although attenuation correction has been in clinical use since the early 1990s and attenuation correction with CT has been available since at least 2005, their publication contributes to the body of knowledge in several important ways. $^{2}$ Very few previous studies have evaluated attenuation correction for thallium imaging. Although attenuation for MPI performed with Tc-99m radioisotopes is common, the lower energy, multiple photo peaks, and greater scatter of Tl-201 make attenuation correction more challenging. Also, few previous studies have been performed in Asian populations which tend to have fewer obese patients and less soft tissue attenuation than the US clinical populations do. Indeed, in the

Reprint requests: Randall C. Thompson, MD, FASNC, Saint Luke's Mid America Heart Institute and The University of MissouriKansas City, 4330 Wornall Rd, Suite 2000, Kansas City, MO 64111; rthompson@saint-lukes.org

J Nucl Cardiol 2019;26:1596-8.

1071-3581/ $\$ 34.00$

Copyright (c) 2018 American Society of Nuclear Cardiology.
Huang study, the average BMI was 25.9, considerably slimmer than the mean BMI ranging from 30 to 33 for patients undergoing SPECT MPI imaging at the Mid America Heart Institute and other US laboratories in recent years. ${ }^{3}$ The study by Huang et al. found that attenuation correction of Tl-201SPECT MPI with a single CT scan improved specificity, especially in men, and especially in the obese, as has generally been the case with other publications on attenuation correction for SPECT. ${ }^{2,4}$ Although thallium is used infrequently in the United States and Europe and the radiation dosimetry is not consistent with ASNC guidelines for radiation reduction, thallium is still used around the world because of its low cost and high availability. ${ }^{5}$ The study by Huang et al. demonstrates that when thallium is used as the radiotracer for myocardial perfusion imaging, CT attenuation correction can improve accuracy, even in a study population that is not particularly obese by the US standards.

In the study by Huang et al., the attenuation-corrected and nonattenuation-corrected images were read blindly, and the demonstrated improvement in accuracy is almost certainly an underestimation. In clinical practice, interpreting physicians review the CT transmission scan for coronary calcifications, thoracic aortic calcification, and other diagnoses. Even when a separate coronary artery calcium score is not obtained, coronary calcifications can be seen, and the severity can be estimated from the CT transmission map. ${ }^{6}$ The presence of these coronary calcifications has been shown to improve the interpretive confidence and the prognostic value of the SPECT studies. ${ }^{7}$ Also, and appropriately, readers routinely adjust their interpretation to incorporate all relevant data. For example, an MPI study of an obese patient with a low pretest likelihood of disease might be "read for specificity", and that of a symptomatic patient with high pretest likelihood will be "ready for sensitivity.' Likewise, the presence of heavy coronary calcifications on a CT transmission map will tend to lead 
Table 1. Value of CT Transmission image in SPECT-CT

\begin{tabular}{l}
\hline Attenuation correction for SPECT MPI with TC-99m \\
based radiopharmaceuticals' \\
Attenuation correction for SPECT MPI with TI-201 \\
radiopharmaceutical \\
Identification of coronary and thoracic aortic \\
atherosclerotic calcifications \\
Identification of other relevant cardiovascular \\
diagnoses \\
Aortic aneurysms \\
Cardiac chamber enlargement \\
Pulmonary artery dilation \\
Pericardial calcifications \\
Pericardial effusions \\
Pleural calcifications \\
Pleural effusions \\
Elevated/paralyzed hemidiaphram \\
Pneumonia/pneumonitis \\
Infiltrative lung diseases \\
Emphysematous lung changes \\
Lung masses \\
Large hiatal hernias and other esophageal diseases
\end{tabular}

a reader to interpret more sensitively, and the absence of any coronary or thoracic aortic calcification's will lead the reader to lean toward specificity. With SPECT-CT instrumentation, if a formal coronary artery calcium score is also obtained during the examination [a process that takes an extra minute or two and a small amount of additional radiation], the diagnostic and prognostic value of the study is enhanced even more. ${ }^{8,9}$

In addition to the benefits of the CT transmission scan for attenuation correction that was demonstrated by Huang et al., and in addition to the diagnostic contributions of identifying vascular calcifications, the CT transmission map has additional diagnostic value that is infrequently discussed. There are at least a dozen relevant cardiovascular diagnoses which can be identified on the transmission map, diagnoses that may explain why the patient had chest pain or shortness of breath that led to the study being ordered in the first place. ${ }^{10}$ Some of these diagnoses are listed in Table 1. In this era when most laboratories find less-frequent myocardial ischemia than in the past, it is all the more attractive to be able to identify findings that might explain a patient's symptoms. For example, dilated pulmonary arteries (that may signify pulmonary hypertension), very large hiatal hernias (which may explain chest pain or shortness of breath), pericardial thickening and calcifications, pleural calcifications, and various lung findings can be very helpful for patient management. Even more frequently, the identification of atherosclerosis by the presence of coronary and thoracic aortic calcification changes treatment algorithms and follow-up for patients that have normal perfusion. ${ }^{11}$

Huang and colleagues have contributed to the body of knowledge regarding the utility of using CT attenuation for thallium SPECT MPI. Although the use of thallium is generally discouraged by ASNC guidelines for minimizing radiation dose, when it is used, either because of cost, availability, or niche indications, CT attenuation can improve the accuracy, especially in males and in obese patients. The other advantages afforded by the concomitant CT imaging are significant and also deserve consideration.

\section{Disclosures}

Author has nothing to disclose.

\section{References}

1. Huang JY, Yen RF, Lee WC, Huang, CK, Hsu PY, Cheng, MF, $\mathrm{Lu}, \mathrm{CC}$, Lin $\mathrm{YH}$, Chien $\mathrm{KL}, \mathrm{Wu}, \mathrm{YW}$. Improved diagnostic accuracy of thallium -201 myocardial perfusion single-photon emission computed tomography with CT attenuation correction. J Nucl Cardiol 2018.

2. Huang JY, Huang CK, Yen RF, Wu HY, Tu YK, Cheng MF, et al. Diagnostic performance of attenuation-corrected myocardial perfusion imaging for coronary artery disease: a systematic review and meta-analysis. J Nucl Med 2016;57:1893-8.

3. Thompson RC, O'Keefe JH, McGhie AI, Bybee KA, Thompson EC, Bateman TM. Reduction of SPECT MPI radiation dose using contemporary protocols and technology: results in 18,000 patients over 8 years. JACC CV Imaging 2018;11:282-3. https://doi.org/10. 1016/j.jcmg.2017.03.008.

4. Thompson R, Heller G, Johnson L, Case J, Cullom S, Garcia E, et al. Value of attenuation correction on ECG-gated SPECT myocardial perfusion imaging related to body mass index. J Nucl Cardiol 2005;12:195-202.

5. Cerqueira MD, Allman KC, Ficaro EP, Hansen CL, Nichols KJ, Thompson RC, Van Decker WA, Yakovlevitch M. Recommendations for reducing radiation exposure in myocardial perfusion imaging. J Nucl Cardiol 2010;17:709-18.

6. Einstein AJ, Johnson LL, Bokhari S, Thompson RC, et al. Agreement of visual estimation of coronary artery calcium from low-dose CT attenuation correction scans in hybrid PET/CT and SPECT/CT with standard Agatston score. J Am Coll Cardiol 2010;56:1914-21.

7. Patchett ND, Pawar S, Miller EJ. Visual identification of coronary calcifications on attenuation correction CT improves diagnostic accuracy of SPECT/CT myocardial perfusion imaging. J Nucl Cardiol 2017;24:711-20.

8. Chang SM, Nabi F, Xu J, Pratt CM, Mahmarian AC, Frias ME, Mahmarian JJ. Value of CACS compared with ETT and myocardial perfusion imaging for predicting long-term cardiac outcome in asymptomatic and symptomatic patients at low risk for coronary disease: Clinical implications in a multimodality imaging world. JACC Cardiovasc Imaging 2015;8:134-44. https://doi.org/ 10.1016/j.jcmg.2014.11.008. 
9. Engbers EM, Timmer JR, Ottervanger JP, Mouden M, Knollema S, Jager PL. Prognostic value of coronary artery calcium scoring in addition to single-photon emission computed tomographic myocardial perfusion imaging in symptomatic patients. Circ Cardiovasc Imaging 2016. https://doi.org/10.1161/CIRCIMAGING. 115.003966

10. Osman MM, Cohade C, Fishman EK, Wahl RL. Clinically significant incidental findings on the unenhanced CT portion of PET/
CT studies: Frequency in 250 patients. J Nucl Med 2005;46:13525 .

11. Bybee KA, Lee J, Markiewicz R, Longmore R, McGhie AI, O'Keefe JH, Hsu BL, Kennedy K, Thompson RC, Bateman TM. Diagnostic and clinical benefit of combined coronary calcium and perfusion assessment in patients undergoing PET/CT, myocardial perfusion stress imaging. J Nucl Cardiol 2010;17:188-96. 